CCR1, CCR2b or CCR3, but the ability of these receptors to allow viral replication in the absence of CCR5 appears to be poor ${ }^{5}$. Alternatively, this person may have been infected by a $\mathrm{T}$ cell-tropic or dual tropic virus, bypassing the requirement for a functional CCR5 molecule. We are currently investigating the cellular tropism of his virus and the ability of lab-adapted strains of HIV to grow in isolated cell populations. This should help clarify the role played by CCR5 and other molecules as co-receptors in primary HIV infection.

In the meantime whereas published reports suggest that lack of CCR5 is highly protective against sexual transmission of
HIV, infection of this individual clearly suggests that CCR5 is not essential as a coreceptor for all primary HIV-infection. Whereas the biological significance of this observation is still unclear, the clinical implications for those known to be homozygous CCR5 $\Delta 32$ are clear.

\section{ROBYN BITI, ROSEMARY FFRENCH,}

Judy Young, BRUCE BenNeTtS \&

GRAEME STEWART

Department of Clinical Immunology, Westmead Hospital, Westmead NSW 2145 Australia

\section{TONG LIANG}

Nepean Sexual Health \& HIV Services, Nepean
Hospital, Penrith NSW 2751, Australia

1. Iluang, Y. et al. The role of a mutant CCR5 allele in HIV-1 transmission and disease progression. Nature Med 2, 1240-1243 (1996).

2. Dean, M. et al. Genetic restriction of HIV-1 infection and progression to AIDS by a deletion allele of the CCR5 structural gene. Science 273, 1856-1862 (1996).

3. Liu, R. et al. Homozygous defect in HIV-1 coreceptor accounts for resistance of some multiply-exposed individuals to HIV-1 infection. Cell 86, 367-377 (1996).

4. Samson, M. et al. Resistance to HIV-1 infection in caucasian individuals bearing mutant alleles of the CCR-5 chemokine receptor gene. Nature 382, 722-725 (1996).

5. Zhang, L., Huang, Y., He, T., Cao, Y. \& Ho, D. HIV-1 subtype and second-receptor use. Nature 383, 768 (1996).

\title{
Stress and the blood brain barrier
}

To the editor - In the December issue, Friedman and colleagues reported that in an experimental animal model for coldinduced stress, the dose of pyridostigmine necessary to inhibit brain acetylcholinesterase by $50 \%$ was $1 / 100$ th that required in non-stressed animals ${ }^{1}$. These results provided a clue to the increased frequency of central nervous system symptoms following pyridostigmine ingestion by 213 Israeli soldiers during the Persian Gulf war ${ }^{2}$ in contrast to the experience of relatively non-stressed volunteers in peacetime ${ }^{3}$. In an accompanying News \& Views ${ }^{4}$, Hanin did not discuss the cause of the increased blood-brain barrier (BBB) permeability associated with stress. We have performed several studies aimed at clarifying the modulatory effects of central adrenergic mechanisms on the BBB and have reported: an amitriptyline-induced increase in brain capillary endothelial cell pinocytosis; ; in increase in BBB permeability following intracerebroventricular administration of catecholamines ${ }^{6}$ and an increase in permeability after electrical stimulation of locus coeruleus, in rats. In vitro studies have also documented changes in brain microvessel endotheilial cell monolayer permeability after exposure to adrenergic drugs $^{8}$. Catecholamines being well known stress hormones, this data sheds some light on the mechanism implicated in stress-induced increased permeability of the blood brain barrier.

ISABEL AZEVEDO \&

ANTONIO SARMENTO
Institute of Pharmacology and Therapeutics,

Faculty of Medicine, 4200-Porto, Portugal

1. Friedman, A. et al. Pyridostigmine brain penetration under stress enhances neuronal excitability and induces early immediate trascriptional response. Nature Med. 2, 1382-1385 (1996).

2. Sharabi, Y. et al. Survey of symptoms following intake of pyridostigmine during the Persian Gulf War. Isr. J. Med. Sci. 27, 656-658 (1991).

3. Glickson, M. et al. The influence of pyridostigmine on human neuromuscular functions - studies in healthy human subjects. Fundam. Appl. Toxicol. 16, 288-298 (1991).

4. Hanin, I. The Gulf War, stress and a leaky blood - brain barrier. Nature Med. 2, 1307-1309 (1996).

5. Sarmento, A., Albino-Teixeira, A. \& Azevedo, I. Amitriptyline-induced morphological alterations of the rat blood - brain barrier. Eur. I. Pharmacol. 176, 69-74 (1990).

6. Sarmento, A., Borges, N. \& Azevedo, I. Adrenergic influences on the control of the blood-brain barrier permeability. Naunyn-Schmiedeberg's Arch. Pharmacol. 343, 633-637 (1991).

7. Sarmento, A., Borges, N. \& Lima, D. Influence of electrical stimulation of locus coeruleus on the rat blood-brain barrier permeability to sodium fluorescein. Acta. Neurocirurgica. 127, 215-219 (1994).

8. Borges, N., Shi, F., Azevedo, I. \& Audus, K.L. Changes in brain microvessel endothelial cell monolayer permeability induced by adrenergic drugs. Eur. J. Pharmacol. 269, 243-248 (1994).

\section{Tumor cells induce apoptosis in lymphocytes}

To the editor - In the December issue of Nature Medicine, Strand et al. ${ }^{1}$ studied the CD95 (Apo-1 or Fas) receptor/ ligand system (which plays an important role in Band T-lymphocyte development and maturation) in patients with hepatocellular cancer (HCC). In all 22 liver tissues examined, they found absence or only focal expression of CD95 in the tumor but homogeneously positive expression in non-tumor tissue. The authors postulate that HCCs seem to evade lymphocytic killing by down-regulating the tumour cell CD95 receptor expression, and envisaged a new mechanism of immune evasion: the active destruction of $\mathrm{T}$ lymphocytes by tumor cells expressing CD95 ligand.
This suggested novel mechanism by which tumors evade the immune system challenges the traditional and generally accepted mechanism of cell defense which proposes that committed lymphocytes destroy tumor cells. The latter mechanism of cytotoxicity has been considered of such biological significance that immunotherapeutic strategies to enhance the number of tumor-specific and committed lymphocytes are being developed. Yet Strand et al. show that the events in HCCs may be otherwise, with tumour cells effecting the killing of lymphocytes.

Working with flat and exophytic adenomas of the colorectal mucosa in humans $^{2}$, we have made observations that seem to substantiate the conclusions of 